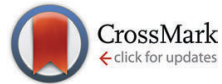

Cite this: J. Mater. Chem. C, 2017, 5, 1553

\section{Correction: Preparation of multi-layer graphene on nickel-coated silicon microchannel plates by a hydrothermal carbonization procedure and its improved field emission properties}

\author{
Dajun Wu, ${ }^{a}$ Chi Zhang, ${ }^{a}$ Cheng Liang, ${ }^{a}$ Yiping Zhu, ${ }^{\star a}$ Shaohui $X u,{ }^{\text {ab }}$ Dayuan Xiong, ${ }^{a}$ \\ Shaolin Xue, ${ }^{c}$ Lianwei Wang ${ }^{* a b}$ and Paul K. Chu ${ }^{b}$ \\ Correction for 'Preparation of multi-layer graphene on nickel-coated silicon microchannel plates by a \\ hydrothermal carbonization procedure and its improved field emission properties' by Dajun Wu et al.,
}

DOI: $10.1039 / \mathrm{c} 7 \mathrm{tc} 90018 \mathrm{j}$ rsc.li/materials-c

The authors regret their oversight in omitting to attribute Fig. 2 in the above paper to their previously reported work in ref. 1. The corrected caption is shown below.
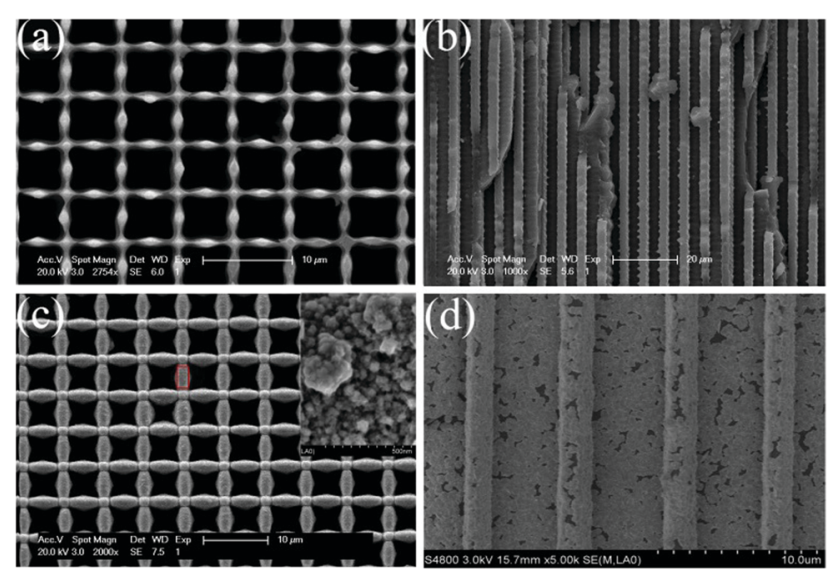

Fig. 2 SEM images: (a) Top surface of Si-MCPs. (b) Cross-sectional morphology of a. (c) Top surface of Ni/Si-MCPs with the inset showing the magnified selected area of Ni/Si-MCPs. (d) Cross-sectional morphology of the Ni/Si-MCPs. Fig. 2(a-c) were reproduced from ref. 1.

The Royal Society of Chemistry apologises for these errors and any consequent inconvenience to authors and readers.

\title{
References
}

1 D. Wu, S. Xu, M. Li, C. Zhang, Y. Zhu, Y. Xu, W. Zhang, R. Huang, R. Qi, L. Wang and P. K. Chu, J. Mater. Chem. A, 2015, 3, 16695-16707.

\footnotetext{
${ }^{a}$ Key Laboratory of Polar Materials and Devices, Ministry of Education, and Department of Electronic Engineering, East China Normal University, 500 Dongchuan Road, Shanghai 200241, P. R. China. E-mail: ypzhu@ee.ecnu.edu.cn; Fax: +86-21-54345119; Tel: +86-21-54342501, +86-21-54345160

${ }^{b}$ Department of Physics and Material Science, City University of Hong Kong, Tat Chee Avenue, Kowloon, Hong Kong, China

${ }^{c}$ College of Science, Donghua University, Shanghai 201620, P. R. China
} 